\title{
ORIGINAL
}

\section{Neurally adjusted ventilatory assist in acute respiratory failure: a randomized controlled trial}

\author{
Robert M. Kacmarek ${ }^{1,2^{*}}$, Jesús Villar ${ }^{3,4^{*}}$ (D) Dácil Parrilla ${ }^{5}$, Francisco Alba ${ }^{6}$, Rosario Solano ${ }^{7}$, Songqiao Liu ${ }^{8}$, \\ Raquel Montiel ${ }^{5}$, Jesús Rico-Feijoo ${ }^{9}$, Anxela Vidal ${ }^{10}{ }^{\text {, Carlos Ferrando }}{ }^{3,11,17}$, Isabel Murcia ${ }^{12}$, Ruth Corpas ${ }^{6}$, \\ Elena González-Higueras ${ }^{7}$, Qin Sun ${ }^{8}$, César E. Pinedo ${ }^{5}$, David Pestaña ${ }^{13}$, Domingo Martínez ${ }^{14}$, César Aldecoa ${ }^{9}$, \\ José M. Añón ${ }^{3,15}$, Marina Soro ${ }^{11}$, Jesús M. González-Martín ${ }^{16}$, Cristina Fernández ${ }^{4}$ and Rosa L. Fernández ${ }^{3,4}$ on \\ behalf of NAVa In Acute respiraTORy failure (NAVIATOR) Network
}

(c) 2020 Springer-Verlag GmbH Germany, part of Springer Nature

\begin{abstract}
Purpose: We hypothesized that neurally adjusted ventilatory assist (NAVA) compared to conventional lung-protective mechanical ventilation (MV) decreases duration of MV and mortality in patients with acute respiratory failure (ARF).

Methods: We carried out a multicenter, randomized, controlled trial in patients with ARF from several etiologies. Intubated patients ventilated for $\leq 5$ days expected to require $\mathrm{MV}$ for $\geq 72 \mathrm{~h}$ and able to breathe spontaneously were eligible for enrollment. Eligible patients were randomly assigned based on balanced treatment assignments with a computerized randomization allocation sequence to two ventilatory strategies: (1) lung-protective MV (control group), and (2) lung-protective MV with NAVA (NAVA group). Allocation concealment was maintained at all sites during the trial. Primary outcome was the number of ventilator-free days (VFDs) at 28 days. Secondary outcome was all-cause hospital mortality. All analyses were done according to the intention-to-treat principle.

Results: Between March 2014 and October 2019, we enrolled 306 patients and randomly assigned 153 patients to the NAVA group and 153 to the control group. Median VFDs were higher in the NAVA than in the control group (22 vs. 18 days; between-group difference 4 days; $95 \%$ confidence interval $[\mathrm{Cl}] 0$ to 8 days; $p=0.016$ ). At hospital discharge, 39 (25.5\%) patients in the NAVA group and 47 (30.7\%) patients in the control group had died (between-group difference $-5.2 \%, 95 \% \mathrm{Cl}-15.2$ to $4.8, p=0.31$ ). Other clinical, physiological or safety outcomes did not differ significantly between the trial groups.
\end{abstract}

Conclusion: NAVA decreased duration of MV although it did not improve survival in ventilated patients with ARF. Keywords: Lung-protective ventilation, Acute respiratory failure, Neurally adjusted ventilatory assist, Intensive care unit, Ventilator-free days, Mortality

\footnotetext{
*Correspondence: rkacmarek@partners.org; jesus.villar54@gmail.com

1 Department of Respiratory Care, Massachusetts General Hospital, 55 Fruit St, Warren 1225, Boston, MA 01460, USA

${ }^{3}$ CIBER de Enfermedades Respiratorias, Instituto de Salud Carlos III,

Madrid, Spain

Full author information is available at the end of the article
}

\section{实




\section{Introduction}

The act of breathing is controlled by the respiratory center in the brainstem, which determines based on input from numerous sites the characteristics of each breath. The respiratory center sends a signal along the phrenic nerve that stimulates the diaphragm muscle cells, causing muscle contraction and descent of the diaphragmatic dome as well as numerous other muscle groups. As a result, the pressure in the airway drops causing an inflow of air into the lungs. Neurally adjusted ventilation assist (NAVA) captures the electrical activity of the diaphragm (Edi), feeds the Edi signal to the ventilator, and uses it to assist the patient's breathing in synchrony with, and in proportion to, the patient's respiratory drive [1-5]. NAVA triggers, cycles and regulates gas delivery based on the diaphragmatic electromyography signal via a specially designed nasogastric tube. As the work of the ventilator and the diaphragm is controlled by the same signal, coupling between the diaphragm and the ventilator is synchronized.

Patient-ventilator asynchrony is a common problem in patients with acute respiratory failure (ARF) receiving mechanical ventilation (MV). Asynchrony has been documented in both volume and pressure assist/ control, as well as pressure support ventilation. Recent data have shown that all patients managed with conventional modes of MV have an asynchrony index $>5 \%$ at various points during the day [6]. Clinicians have a hard time identifying the presence of asynchrony at the bedside [7] despite that numerous ineffective triggering has been associated with increased duration of mechanical ventilation [8]. Previous trials comparing pressure support ventilation with NAVA [1,9-12] did not provide sufficient evidence for duration of MV or survival benefits in patients with ARF. We hypothesized that NAVA compared to conventional lung-protective MV would improve patient-ventilator interaction by making the transition to spontaneous breathing much quicker and easier, and would result in an increase of VFDs, a decrease in ventilator-associated complications, and in a better survival. The goal of this study was to compare outcomes with NAVA versus conventional lung-protective $M V$ in patients with ARF who are expected to require ventilatory support for at least $72 \mathrm{~h}$. The primary outcome variable was VFDs. Secondary outcomes of interest were allcause ICU, hospital and 90-day mortality after randomization, duration of MV in survivors, reintubation rate, and pulmonary complications.

\section{Take-home message}

In this randomized clinical trial in 306 patients with ARF from several etiologies, NAVA ventilation was significantly associated with increased ventilator-free days and decreased duration of MV in ICU survivors, when compared to conventional lung-protective MV. Whether these benefits can be translated into better hospital survival in some specific etiologies of ARF, remains to be determined in future trials.

\section{Methods}

\section{Study design}

This study was a multicenter, randomized, controlled trial conducted in 15 centers (14 in Spain and 1 in China), and designed in accordance with the fundamental principles of the Declaration of Helsinki [13]. The trial protocol and the statistical analysis plan were previously published [14] (available in the Electronic Supplementary Material, ESM). The protocol was approved by the referral Ethics Committee (Hospital Clínico Universitario, Valencia, Spain). Patient representatives provided written informed consent for inclusion into the trial. A data and safety monitoring board (DSMB) oversaw conduct of the trial, while remaining blind to the outcomes of interest. The study conformed to current guidelines for randomized clinical trials [15].

\section{Patient population and randomization}

Patients were eligible for inclusion if they met the following criteria during screening: age $\geq 18$ years, hypoxemic $\left(\mathrm{PaO}_{2} / \mathrm{FiO}_{2}\right.$ ratio $<300 \mathrm{mmHg}$ in a patient without chronic pulmonary disease) or hypercapnic $\left(\mathrm{PaCO}_{2}>45 \mathrm{mmHg}\right.$ in a patient with chronic pulmonary disease, $\mathrm{pH}<7.35$ in a patient with chronic pulmonary disease) ARF, intubated and mechanically ventilated for $\leq 5$ days but expected to be ventilated for $\geq 72 \mathrm{~h}$, and be able to trigger almost every mechanical breath. Patients were excluded from enrollment if they met any of the following conditions: moderate or severe acute respiratory distress syndrome [16], required noninvasive ventilation at the time of screening, were unable to breathe spontaneously, had neuromuscular or neurological disease, had any esophageal medical/surgical contraindication that might be traumatized by the insertion of the NAVA catheter, were pregnant, had body weight less than $35 \mathrm{~kg}$, lack of informed consent, or had a poor short-term prognosis (as defined by a high risk of death within three months), and had other undefined reason for not participating.

Eligible, informed consented patients were randomized into two arms: (1) conventional lung-protective MV (control group) or (2) NAVA. Randomization was based 
on a 1:1 ratio and stratified by centers using a computergenerated assignment sequence with a variable block size of prenumbered, opaque, sealed envelopes sent to each participating ICU. There was no limitation in the number of enrolled patients per center during the trial except the availability of the Servo-i ventilator (Maquet, Sölna, Sweden). Patients, investigators and attending clinicians were unaware of the sequence of patients in each treatment arm from those blocks. The trial characteristics did not allow the blinding of investigators to the intervention being tested.

\section{Trial interventions}

Patients in the control group were ventilated using either volume assist/control $(\mathrm{A} / \mathrm{C})$, pressure assist/control $(\mathrm{A} / \mathrm{C})$, pressure support $(\mathrm{PS})$, or pressure-regulated volume control (PRVC), at the discretion of the medical team, using tidal volumes (VT) in the range of 4-8 $\mathrm{ml} /$ $\mathrm{kg}$ predicted body weight (PBW) and plateau pressure or pressure (control or support) level setting of $\leq 30 \mathrm{cmH}_{2} \mathrm{O}$. During $\mathrm{A} / \mathrm{C}$ the backup rate was set to ensure that the vast majority of the breaths were patients triggered.

Patients in the NAVA group were ventilated with Servo-i ventilators. We used one size NAVA catheter (Edi catheter $16 \mathrm{Fr}$.) in all patients. Placement of the NAVA catheter is similar to the placement of a typical nasogastric tube; placement was achieved in all patients. Details for positioning the Edi catheter, initial NAVA settings, and subsequent adjustment of NAVA, are published in the original protocol [14]. The NAVA level was set initially at zero, then the maximum Edi was determined as the average level over the next three to five breaths without ventilatory support with $5 \mathrm{cmH}_{2} \mathrm{O}$ of positive endexpiratory pressure (PEEP). The actual NAVA level was then titrated by the clinician to achieve: (1) an Edi equal to approximately $50 \%$ of the maximum Edi, (2) an average VT of between 4 and $8 \mathrm{ml} / \mathrm{kg}$ PBW, and (3) an average respiratory rate between 15 and 40 per min. During initial setting of NAVA, $\mathrm{PaCO}_{2}$ occasionally increased to insure appropriate Edi activity. In addition, the trigger sensitivity was set as sensitive as possible without causing auto-triggering and the maximum pressure limit in NAVA was set at $40 \mathrm{cmH}_{2} \mathrm{O}$. The NAVA catheter as recommended by the manufacturer was changed every 5 days. As during the initial setting of NAVA, the maximum Edi was determined daily (see ESM). Maquet supplied the NAVA catheters, but the company had no role in the trial design, data analysis, or data interpretation.

Minimal sedation and appropriate analgesia were maintained in all patients. Weaning was performed by a spontaneous breathing trial (SBT) $[14,17]$. Prior to starting SBT the patient had: (1) a partial reversal of the underlying cause of ARF, (2) $\mathrm{SpO}_{2}>88 \%$ or $\mathrm{PaO}_{2}>55 \mathrm{mmHg}$ with $\mathrm{FiO}_{2} \leq 0.4$ and $\mathrm{PEEP} \leq 8 \mathrm{cmH}_{2} \mathrm{O}$, (3) hemodynamic stability, (4) a level of sedation appropriate for SBT, and (5) the ability to breathe spontaneously. The SBT was conducted for at least $30 \mathrm{~min}$ and no longer than $120 \mathrm{~min}$, and tested with a T-piece or with pressure support at $8 \mathrm{cmH}_{2} \mathrm{O}$ with the same $\mathrm{FiO}_{2}$ as during $\mathrm{MV}$. The SBT could be repeated each day. If the patient passed the SBT, a decision for extubation (or disconnection of MV from tracheostomy) was taken, unless there was a reason not to extubate or reconnect.

\section{Outcomes measures}

The primary outcome was the number of VFDs, defined as the number of days alive and free from MV from randomization and initiation of treatment to day- 28 after randomization. For calculating VFDs, we made the following considerations: (1) successful liberation from MV should last $>48 \mathrm{~h}$ without reintubation in a 28-day survivor; (2) extubations only count from the last successful extubation within a 28-day survivor; (3) VFDs were awarded zero days if the patient was ventilated for $\geq 28$ days or died before 28 days (irrespective of intubation status). Secondary outcomes included all-cause ICU, hospital and mortality at 90 days after randomization, duration of MV in survivors, reintubation rate, and pulmonary complications (see ESM).

\section{Statistical analysis}

For sample size calculations, we estimated that the average patient would have 21 VFDs with a standard deviation of 6 VFDs, based on Wit et al. [8]. We estimated that NAVA will increase VFDs by 2 days. Power analysis was performed according to Schoenfeld et al. [18]. With an alpha of 0.05 and a power of $80 \%$, we estimated a sample size of 306 patients (153 in each group). Temporal changes in organ dysfunction was assessed by the Sequential Organ Failure Assessment (SOFA) scale [19].

Descriptive statistics are expressed as mean \pm standard deviation (SD), median and $25 \%$ and $75 \%$ percentiles $\left(\mathrm{P}_{25}-\mathrm{P}_{75}\right)$, frequency, and percentages, depending on the nature and distribution of variables. Variables normally distributed were compared with the Student's t-test. For variables without a normal distribution, the Mann-Whitney U-rank test was used for comparison. Categorical variables were compared using Fisher's exact test. Primary and secondary outcomes are reported with between-groups observed differences and 95\% confidence intervals (CI). The $95 \%$ CI for the difference between medians for VFDs in both groups was estimated using a bootstrap procedure (10,000 replications). Analysis was performed according to the intention-to-treat principle, without adjustment for multiple comparisons. We analyzed time to death and probability of successful 
weaning to day-90 after randomization in both groups using Kaplan-Meier curves. A two-sided $p$ value of $<0.05$ indicated statistical significance. All statistical analyses were performed with R software (R Core Team 2019).

\section{Results}

\section{Patient characteristics}

From March 2014 through October 2019, a total of 310 patients were enrolled: 155 assigned to the control group and 155 to the NAVA group. One patient in each arm was lost to follow-up due to transferring to other hospitals, and one patient in each arm withdrew the consent after being enrolled. As a result, analysis was performed in 153 patients in each arm, as planned (Fig. 1). Most common reasons for exclusion before enrollment were moderate/severe acute respiratory distress syndrome, postsurgical patients requiring short $\mathrm{MV}$, and severe neuromuscular or neurological disease. Twenty-three

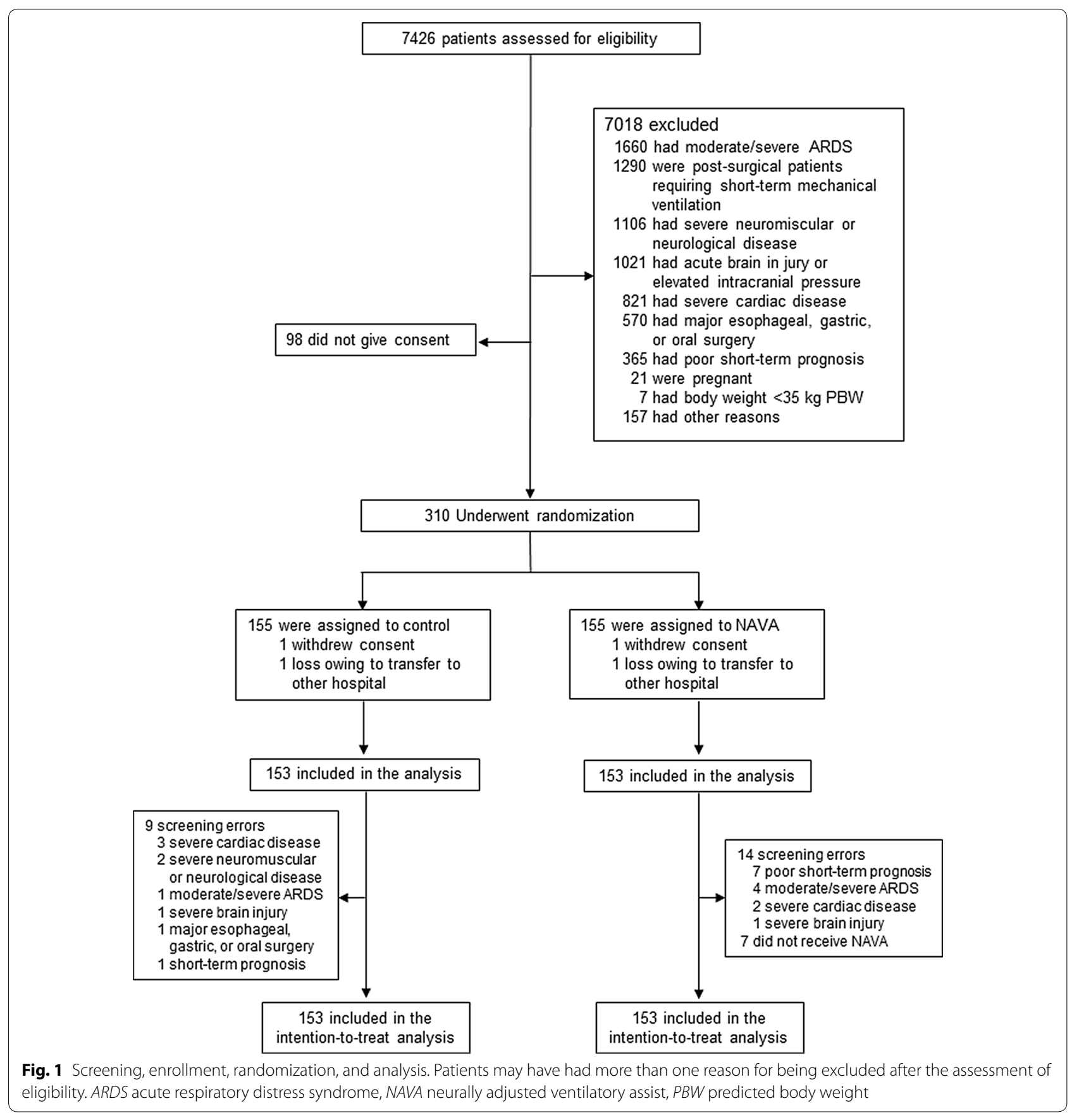


patients (14 in the NAVA group and 9 in the control group) were enrolled despite having exclusion criteria, and 7 patients in the NAVA group did not receive the intervention. Median enrollment across participating sites was 18 patients $\left(\mathrm{P}_{25}-\mathrm{P}_{75}: 4-30\right.$ patients). During the study period (Table S1), seven centers abandoned the trial for reasons unrelated to the study. Enrollment into the trial was performed at $2.2 \pm 1.5$ days after the initiation of MV. The time between intubation and enrollment was the same for both groups. Baseline characteristics at randomization did not differ between groups (Table 1). Main primary diagnoses were pneumonia, sepsis, and postsurgical patients.

\section{Outcomes}

In the intention-to-treat analysis, patients in the NAVA group had a higher median VFDs than patients in the control group (22 vs. 18 days, between-groups difference 4 days, $95 \%$ CI 0 to 8 days; $p=0.016$ ) (Table 2). All-cause ICU and hospital mortality, and mortality at 90 days after randomization did not differ significantly between the two groups (Table 2, Fig. 2).

Actual duration of $\mathrm{MV}$ in ICU survivors was shorter in the NAVA group than in control group $(6.7 \pm 7.7 \mathrm{vs}$. $10.0 \pm 12.9$ days; between-group difference 3.3 days, $95 \%$ CI $0.61-5.97 ; p=0.016)$. Within the 28 days after randomization, $11.1 \%$ of patients (17 of 153 ) in the NAVA

\section{Table 1 Baseline characteristics of $\mathbf{3 0 6}$ patients with acute respiratory failure at randomization}

\begin{tabular}{|c|c|c|}
\hline Characteristics & NAVA group $(N=153)$ & Control group $(N=153)$ \\
\hline Age, years & $63.9 \pm 15.4$ & $64.7 \pm 14.1$ \\
\hline Male gender, $N(\%)$ & $100(65.4)$ & $101(66)$ \\
\hline \multicolumn{3}{|l|}{ Primary diagnoses, $N(\%)$} \\
\hline Pneumonia & $49(32)$ & $49(32)$ \\
\hline Sepsis & $40(26.1)$ & $36(23.5)$ \\
\hline Post-surgical & $22(14.4)$ & $18(11.8)$ \\
\hline Chronic obstructive pulmonary disease & $15(9.8)$ & $18(11.8)$ \\
\hline Acute pancreatitis & $5(3.3)$ & $4(2.6)$ \\
\hline Aspiration & $4(2.6)$ & $4(2.6)$ \\
\hline Overdose/poisoning & $4(2.6)$ & $4(2.6)$ \\
\hline Trauma & $3(2)$ & $5(3.3)$ \\
\hline Heart failure & $3(2)$ & $4(2.6)$ \\
\hline Others & $8(5.2)$ & $11(7.2)$ \\
\hline APACHE II score & $16.1 \pm 7$ & $16.4 \pm 7.2$ \\
\hline Time from intubation to study inclusion, days & $2.4 \pm 1.5$ & $2 \pm 1.5$ \\
\hline SOFA score & $6.4 \pm 3.1$ & $6.8 \pm 3.3$ \\
\hline Tidal volume, ml/kg PBW & $7.4 \pm 1.4$ & $7.2 \pm 1.2$ \\
\hline Respiratory rate (breaths/min) & $19 \pm 6$ & $19 \pm 5$ \\
\hline Peak inspiratory pressure $\left(\mathrm{cmH}_{2} \mathrm{O}\right)$ & $22 \pm 7$ & $24 \pm 7$ \\
\hline Plateau pressure $\left(\mathrm{cmH}_{2} \mathrm{O}\right)$ & $19 \pm 5(n=105)$ & $20 \pm 5(n=128)$ \\
\hline PEEP $\left(\mathrm{cmH}_{2} \mathrm{O}\right)$ & $8 \pm 2$ & $8 \pm 3$ \\
\hline $\mathrm{FiO}_{2}$ & $0.44 \pm 0.09$ & $0.47 \pm 0.12$ \\
\hline $\mathrm{PaO}_{2}(\mathrm{mmHg})$ & $106 \pm 31$ & $109 \pm 36$ \\
\hline $\mathrm{PaO}_{2} / \mathrm{FiO}_{2}(\mathrm{mmHg})$ & $250 \pm 87$ & $244 \pm 88$ \\
\hline $\mathrm{PaCO}_{2}(\mathrm{mmHg})$ & $43.1 \pm 7.6$ & $44.8 \pm 9.8$ \\
\hline $\mathrm{pH}$ & $7.41 \pm 0.09$ & $7.39 \pm 0.08$ \\
\hline \multicolumn{3}{|l|}{ Ventilatory mode, $N(\%)$} \\
\hline Volume control & $54(35.3)$ & $68(44.4)$ \\
\hline Pressure control & $18(11.8)$ & $15(9.8)$ \\
\hline Pressure support & $43(28.1)$ & $27(17.6)$ \\
\hline Pressure-regulated volume control & $38(24.8)$ & $43(28.1)$ \\
\hline
\end{tabular}

Plus-minus values are means \pm SD. Percentages may not total 100 because of rounding. There were no significant differences between the groups. FiO ${ }_{2}$ denotes fraction of inspired oxygen

APACHE Acute Physiology and Chronic Health Evaluation II scores range from 0 to 71, PBW predicted body weight, $P E E P$ positive end-expiratory pressure, SOFA Sequential Organ Failure Assessment score [19] 
Table 2 Primary and secondary outcomes*

\begin{tabular}{|c|c|c|c|c|}
\hline Variables & NAVA group $(N=153)$ & Control group $(N=153)$ & Difference $(95 \% \mathrm{Cl})$ & $p$ value \\
\hline Ventilator-free days median $\left(\mathrm{P}_{25}-\mathrm{P}_{75}\right)$ & $22(3-25)$ & $18(0-24)$ & $4(0 \text { to } 8)^{\natural}$ & 0.016 \\
\hline All-cause ICU deaths N (\%) & $30(19.6)$ & $27(17.6)$ & $2 \%(-6.8$ to 10.7$)$ & 0.66 \\
\hline All-cause hospital deaths N (\%) & $39(25.5)$ & $47(30.7)$ & $5.2 \%(-4.8$ to 15.2$)$ & 0.31 \\
\hline All-cause mortality at 90 days after randomization, N (\%) & $41(26.8)$ & $50(32.7)$ & $5.9 \%(-4.3$ to 16$)$ & 0.26 \\
\hline Total duration of MV in days, mean \pm SD & $7.8 \pm 8.1$ & $11.9 \pm 16.2$ & $-4.1(-7$ to -1.3$)$ & 0.005 \\
\hline Duration of MV in ICU survivors, days, mean \pm SD & $6.7 \pm 7.8(n=123)$ & $10 \pm 13(n=126)$ & $3.3(0.6$ to 6$)$ & 0.0162 \\
\hline Patients with extubation failure, $N(\%)^{\S}$ & $17(11.1)$ & $33(21.6)$ & $10.5 \%(2.2$ to 18.7$)$ & 0.0135 \\
\hline Tracheostomy in ICU, N (\%) & $32(20.9)$ & $32(20.9)$ & $0 \%(-9.1$ to 9.1$)$ & 1 \\
\hline Development of ARDS, $N(\%)^{2}$ & $16(10.5)$ & $19(12.4)$ & $2 \%(-5.3$ to 9.3$)$ & 0.59 \\
\hline Pneumothorax, N (\%) & $3(2)$ & $4(2.6)$ & $0.6 \%(-3.3$ to 4.8$)$ & 0.7 \\
\hline Development of pneumonia, $N(\%)^{\boldsymbol{\alpha}}$ & $4(2.6)$ & $2(1.3)$ & $1.3 \%(-2.4$ to 5.3$)$ & 0.41 \\
\hline
\end{tabular}

* No missing data were observed for patients' outcomes. ARDS acute respiratory distress syndrome. $C l$ confidence interval, ICU intensive care unit, $M V$ mechanical ventilation, $S D$ standard deviation

9 The width of $\mathrm{Cl}$ for the median difference in ventilator-free days at 28 days should not be used to infer definitive treatment differences

$\S$ Data included the period from randomization to hospital discharge

* Data included the period from randomization to ICU discharge

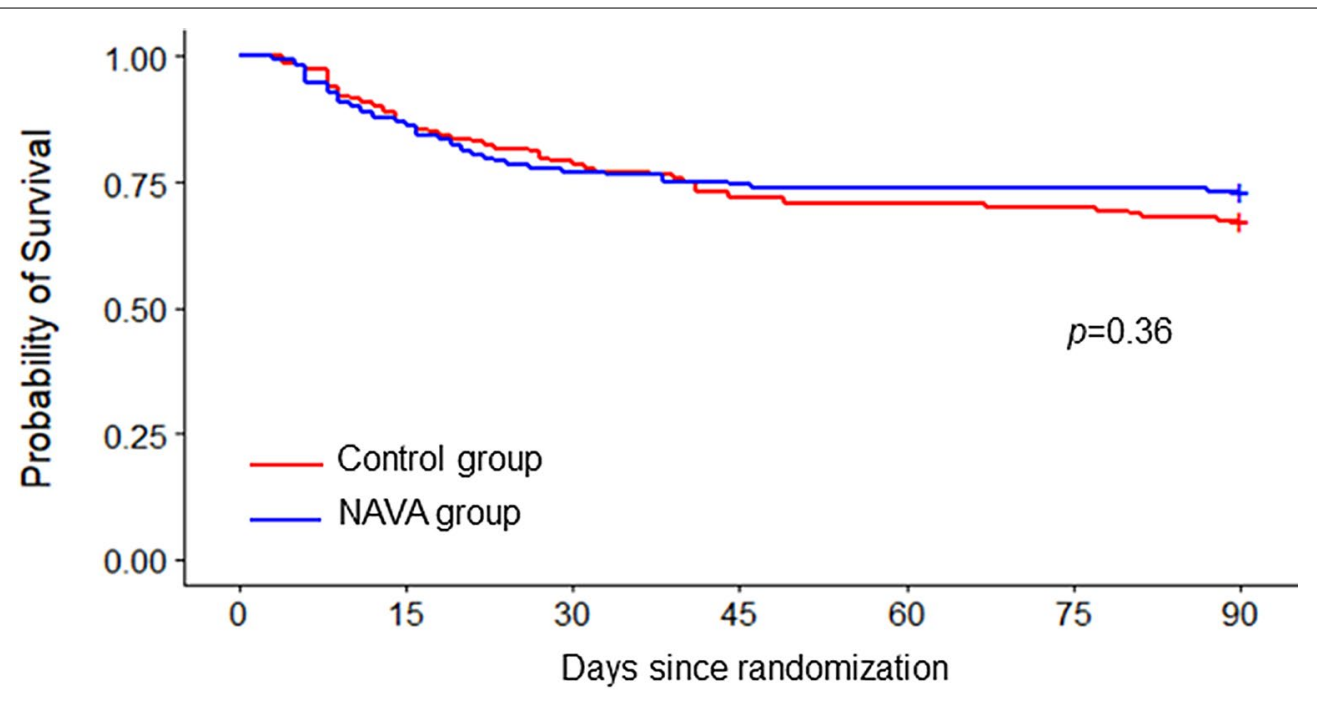

\begin{tabular}{|c|c|c|c|c|c|c|c|}
\hline \multicolumn{8}{|c|}{ Number at risk } \\
\hline Control- & 153 & 133 & 121 & 110 & 108 & 107 & 103 \\
\hline NAVA & 153 & 133 & 118 & 114 & 113 & 113 & 112 \\
\hline & 0 & 15 & 30 & 45 & 60 & 75 & 90 \\
\hline
\end{tabular}

Fig. 2 Kaplan-Meier estimates of survival at 90 days after randomization

group and $21.6 \%$ (33 of 153) in the control group developed extubation failure $(p=0.013)$, and required reintubation or reconnection to MV (in case they had a tracheotomy). In patients with extubation failure, mean duration of MV (15.2 \pm 11.2 vs. $15.3 \pm 10.6$ days) and number of hospital deaths (10/17 vs. 10/33) did not differ significantly between the trial groups. Figure S1 illustrates the estimated Kaplan-Meier curve at 90 days for successful weaning. The decision to perform multiple SBTs in one day was left up to the discretion of the 
managing clinician. After extubation, 37 (24\%) patients in the NAVA group and $46(30 \%)$ patients in the control group required NIV. NIV was used in 7/17 (41\%) of reintubated patients in the NAVA group and 10/33 (30\%) of reintubated patients in the control group $(p=0.53)$.

Occurrence of pneumothorax was similarly distributed in both groups (Table 2). Major causes of ICU death were multisystem organ failure and refractory hypoxemia (Table S2), mostly related to the underlying disease processes. Peak inspiratory pressure was significantly lower in the NAVA group at $24 \mathrm{~h}(p=0.0038)$ and at day $2(p=0.0126)$ after randomization. However, no other physiologic variables varied between groups during the trial (Table 3).

\section{Adverse events}

Rate of serious adverse events related to study protocol did not differ between the trial groups (Table S3). One death resulted from a serious adverse event unrelated to the study protocol (details in ESM).

\section{Discussion}

The primary findings of our study are as follows: (1) The NAVA group had a greater number of VFD's $(22$ VFD's) than the control group (18 VFD's) ( $p=0.016)$; (2) Actual duration of MV in ICU survivors was shorter in the NAVA group $(6.7+7.7$ days $)$ than the control group $10.0+12.9$ days $(p=0.016)$; (3) At 28 days after randomization, $11.1 \%(17 / 153)$ in the NAVA group and $21.6 \%$ $(33 / 153)$ in the control group required reintubation $(p=0.013)$.

Assisted MV is a complex process that requires a close interaction between the ventilator and the patient $[20,21]$. The complexity of this interaction is frequently underappreciated by the bedside clinician. All available studies on NAVA in patients with ARF have limitations regarding the clinical applicability of its theoretical physiological benefits [22,23], mainly because patient populations were heterogeneous in terms of cause of ARF. Ideally under optimal conditions, NAVA has two important features: the delivered pressure in synchrony with the diaphragmatic activity, and the VT controlled by the output of the patient's respiratory center [24]. Since all that is set during NAVA is the proportion of effort provided by the ventilator to supplement the patient's ventilatory drive, for a given NAVA level, the airway pressure varies breath-by-breath in proportion to Edi. Three randomized controlled trials on NAVA have been previously published [9,11,12]. Demoule et al. [9] randomized 128 patients ready for partial ventilatory support to NAVA or pressure support. The percentage of patients remaining on partial ventilatory support for the first $48 \mathrm{~h}$ after randomization was $67.3 \%$ NAVA vs $63.3 \%$ control, VFDs were
21 in NAVA and 17 in control $(p=0.12)$, post-extubation NIV was used in $43.6 \%$ NAVA vs $66 \%$ control $(p<0.001)$. NAVA did not affect mortality. Hadfield et al. [11] randomized 78 patients at risk for prolonged MV to receive NAVA or pressure support. They found that NAVA was associated with more VFDs, however, no significant differences were observed in duration of MV, ICU or hospital stay. Although they also reported fewer hospital deaths in the NAVA group, no significant differences were observed in ICU, 28-day, or 90-day mortality. Most recently, Liu et al. [12] randomized the use of NAVA and pressure support in 99 difficult-to-wean patients. They observed that NAVA reduced the time of weaning and increased VFDs, with no effects on ICU, 28-day, or hospital mortality. Our study primarily differs from these studies in that it accepted patients with ventilatory failure of all types and was used throughout the entire course of a patient's need for ventilatory support. In a previous study, we reported that NAVA, as compared to pressure support ventilation, improved synchrony, reduced ventilatory drive, increased breath-to-breath mechanical variability, and improved patient comfort in 12 mechanically ventilated pediatric patients [25]. In theory, NAVA offers protection against dangerous levels of volume and pressure. Colombo et al. [1] performed a physiologic study in 14 patients comparing NAVA and pressure support ventilation and found that NAVA averted the risk of overassistance and improved patient-ventilator interaction. Our trial was not a physiological study but investigators were encouraged to check routinely the optimal positioning of the NAVA catheter to determine the optimal setting in terms of unloading patient's respiratory muscles. No patient was excluded from the study as a result of an inability to properly place the NAVA catheter. We did not collect information regarding body position and intraabdominal pressure during the trial, but there is evidence suggesting that those factors do not compromise a stable signal of the NAVA catheter if its position is adjusted after changes in ventilator settings, clinical condition, or patient positioning [26]. On average, VT was maintained within the range of lung-protective ventilation $(4-8 \mathrm{ml} /$ $\mathrm{kg}$ PBW) in both trial groups, despite that NAVA generates VTs that are independent of the assist level once the patient's ventilation needs are satisfied [27], and NAVA settings were not adjusted solely on VT or $\mathrm{PaCO}_{2}$ targets. In addition, by protocol plateau pressure was maintained $<30 \mathrm{cmH}_{2} \mathrm{O}$ in both groups while NAVA level was adjusted on a daily level to $50 \%$ of the unassisted Edi.

Ventilatory assistance with NAVA remains under the patient's control but we are unaware of what is the best time to begin NAVA. In our trial, titration of the NAVA level was performed in conjunction with gradual discontinuation of sedative agents. We acknowledge that 


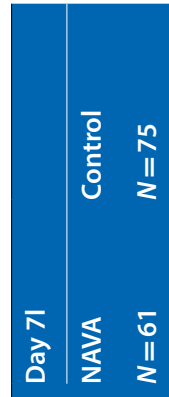

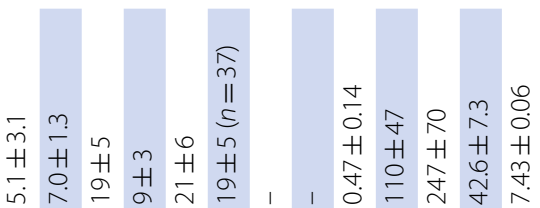

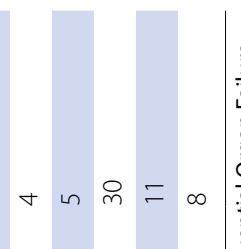

产爱

离

$\rightarrow$ in $\stackrel{m}{=\infty}$

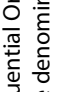

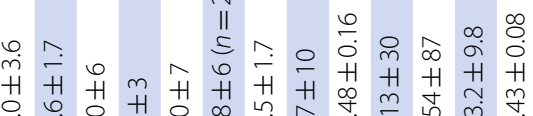

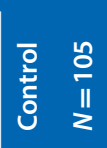

内i

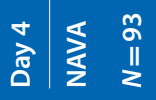

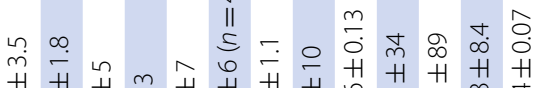

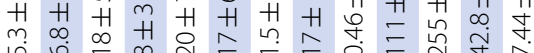

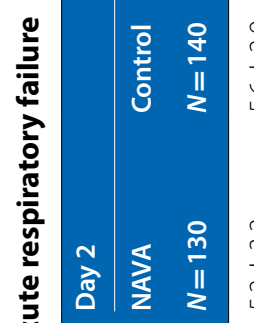

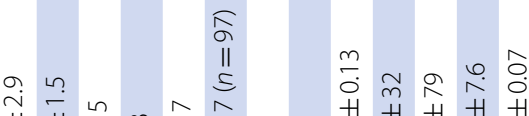

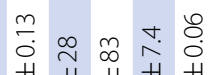

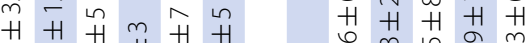

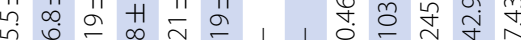

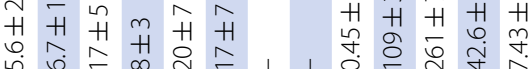

s.

ซु

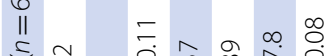

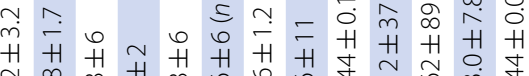

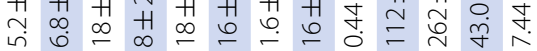

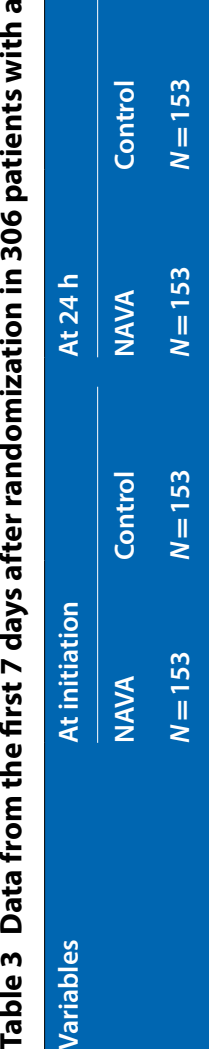

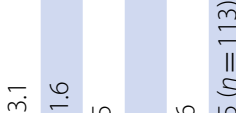

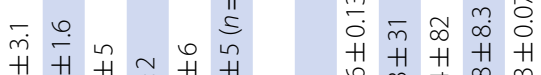

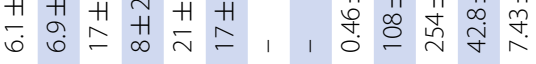

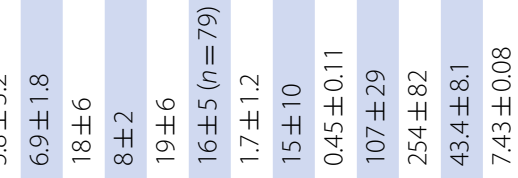

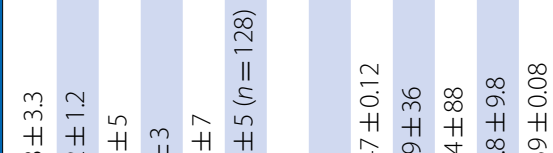

H H H $\begin{aligned} & n-H \\ & 0\end{aligned}$

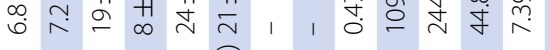

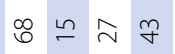

II

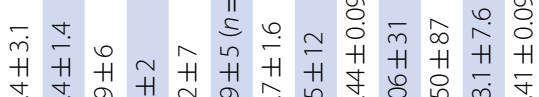

ำ $+\circ \div$

离富

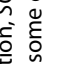

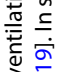

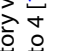

$\because \bullet \stackrel{\infty}{\sim} a$

要

蒂畩兽

劳

离

紊旁

空嘀

혼

穿

흥 응

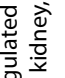

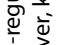

d.

离

눙

$\stackrel{\sim}{\sim}$ 的 $8 \underset{\sim}{m}$ i

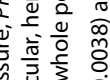

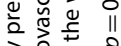

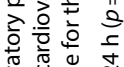

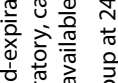

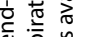

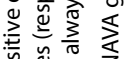

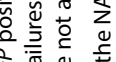

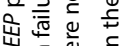

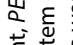

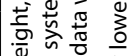

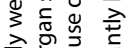

중 읗

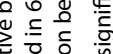

가요

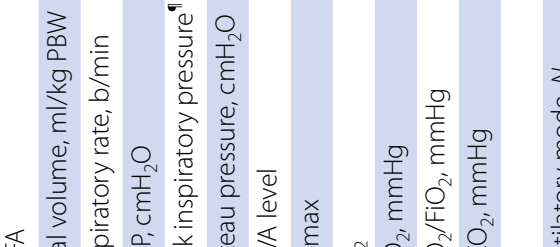


clinicians are accustomed to other modes of MV or to set the pressure support levels for liberating the patient from MV. Initially, clinicians and local investigators involved in the study were uncomfortable with a nasogastric tube that is used for feeding, administration of medication, and for sensing the electrical activity of the diaphragm. Since in NAVA the mechanical support is on-triggered and off-triggered by the Edi, the breathing pattern during NAVA is much less informative for clinicians than other modes of MV for subsequent adjustments. Our trial confirmed that clinicians have a limited accuracy for predicting duration of MV [28]. Mean VFDs in both groups were smaller than expected, probably due to the type of patients enrolled in the trial. Our inclusion criteria specified that expected duration of MV for enrolling a patient should be $\geq 72 \mathrm{~h}$, and we estimated the sample size of the trial based on an average of 21 VFDs. However, the actual duration of MV after randomization in some patients was markedly larger than predicted, which could explain the high proportion of patients with tracheostomy (64/306, 20.9\%). In a multicenter trial comparing NAVA and pressure support ventilation in 128 patients recovering from ARF, investigators reported 21 VFDs in the NAVA group [9]. Of note, the rate of reintubation in the NAVA arm of our trial was relatively low (11.1\%). It is estimated that the reintubation rate after extubation failure for all indications is about $20 \%$ [29]. In a small study with 20 patients comparing NAVA or pressure support ventilation during spontaneous breathing trials, the authors reported an extubation failure rate of $25 \%$, much higher than in our trial [10].

We acknowledge some limitations of this trial. First, the study by its very nature was unblinded, which could have biased the decisions made by caregivers in the management of recruited patient. Second, weaning followed the ARDSnet protocol but the protocol determining when or if a patient should be reintubated could have been more detailed. Third, patient severity of illness was greater than expected in our study population. Fourth, since participating investigators were asked to estimate duration of MV regardless of their probability for survival, it is plausible that they underestimated the true severity of the underlying disease and the likelihood for successful weaning and survival. Fifth, most patients died in the ICU, and the most common causes of death were similar to those from patients with severe acute respiratory failure [30], a scenario that was not anticipated in our trial design. Sixth, the strict inclusion/exclusion criteria for enrolling patients could have biased our results toward excluding difficult-to-wean patients ventilated for more than 5 days. Seventh, we could not be assured that some of the breaths in the control group could have been controlled mechanical breaths, or patients did not initiate breaths because of the function of the modes used. Finally, the diversity of diagnoses could limit the generalization of our findings.

\section{Conclusion}

In conclusion, ventilating patients with NAVA compared with conventional modes of partial ventilatory support increased the number of VFDs in a heterogeneous population of patients with ARF. Whether the theoretical benefits of NAVA can be translated into better survival in some specific etiologies of ARF or in difficult-to-wean patients without setting a specific time-period from the time of intubation remains to be determined in further trials. The unblinded nature of studies such as this always make interpretation of results difficult.

\section{Electronic supplementary material}

The online version of this article (https://doi.org/10.1007/s00134-020-06181-5) contains supplementary material, which is available to authorized users.

\section{Author details}

${ }^{1}$ Department of Respiratory Care, Massachusetts General Hospital, 55 Fruit St, Warren 1225, Boston, MA 01460, USA. ${ }^{2}$ Department of Anesthesia and Critical Care Medicine, Harvard University, Boston, MA, USA. ${ }^{3}$ CIBER de Enfermedades Respiratorias, Instituto de Salud Carlos III, Madrid, Spain. ${ }^{4}$ Research Unit, Hospital Universitario Dr. Negrín, Barranco de la Ballena s/n, 4th Floor South Wing, 35019 Las Palmas de Gran Canaria, Spain. ${ }^{5}$ Intensive Care Unit, Hospital Universitario N.S. de Candelaria, Santa Cruz de Tenerife, Spain. ${ }^{6}$ Intensive Care Unit, Hospital N.S. del Prado, Talavera de La Reina, Toledo, Spain. ${ }^{7}$ Intensive Care Unit, Hospital Virgen de La Luz, Cuenca, Spain. ${ }^{8}$ Intensive Care Unit, Zhongda Hospital, Southeast University, Nanjing, China. ${ }^{9}$ Department of Anesthesia, Hospital Universitario Río Hortega, Valladolid, Spain. ${ }^{10}$ Intensive Care Unit, Hospital Universitario Fundación Jiménez Díaz, Madrid, Spain. ${ }^{11}$ Department of Anesthesia, Hospital Clínico Universitario de Valencia, Valencia, Spain. ${ }^{12}$ Intensive Care Unit, Complejo Universitario de Albacete, Albacete, Spain. ${ }^{13}$ Department of Anesthesia, Hospital Universitario Ramón Y Cajal, IRYCIS, Madrid, Spain. ${ }^{14}$ Intensive Care Unit, Hospital Universitario Virgen de Arrixaca, Murcia, Spain. ${ }^{15}$ Intensive Care Unit, Hospital Universitario La Paz, Madrid, IdiPAZ, Spain. ${ }^{16}$ Biostatistics Division, Research Unit, Universitario Dr. Negrín, Las Palmas, Spain. ${ }^{17}$ Present Address: Department of Anesthesiology and Critical Care, Hospital Clinic, Barcelona, Spain.

\section{Acknowledgements}

All members of the NAVIATOR Network are listed below: Isabel Murcia: Intensive Care Unit, Hospital Universitario de Albacete, Hermanos Falcó 37, 02006 Albacete. Spain; Carlos Ferrando: Postoperative Care Unit, Hospital Clínico Universitario de Valencia, Blasco Ibañez 17, 46010 Valencia. Spain; José Ferreres: Intensive Care Unit, Hospital Clínico Universitario de Valencia, Blasco Ibañez 17, 46010 Valencia. Spain; Anxela Vidal: Intensive Care Unit, Hospital Universitario Jiménez Díaz, Avda. Reyes Católicos 2, 28040 Madrid. Spain; José M. Añón: Intensive Care Unit, Hospital Universitario La Paz, Paseo de la Castellana 261, 28046 Madrid. Spain; Juan A. Soler: Intensive Care Unit, Hospital Universitario Morales Meseguer, Marqués de los Vélez s/n, 30008 Murcia. Spain; Raquel Montiel: Intensive Care Unit, Hospital Universitario N.S. de Candelaria, Carretera del Rosario 145, 38010 Santa Cruz de Tenerife. Spain; Francisco Alba: Intensive Care Unit, Hospital N.S. del Prado, Crta. de Madrid, Km 114, 45600 Talavera de la Reina, Toledo. Spain; José Rubio: Intensive Care Unit, Hospital Puerta del Mar, Avda. Ana de Viya 21, 11009 Cádiz. Spain; David Pestaña: Postoperative Care Unit, Hospital Universitario Ramón y Cajal, Crta. Colmenar Viejo, Km 9.1 28034 Madrid. Spain; Jesús Blanco: Intensive Care Unit, Hospital Universitario Río Hortega, Dulzaina 2, 47012 Valladolid. Spain; César Aldecoa: Postoperative Care Unit, Hospital Universitario Río Hortega, Dulzaina 2, 47012 Valladolid. Spain; Nela Hernández: Intensive Care Unit, Hospital Universitario de Txagorritxu, José Atxotegui s/n, 01007 Vitoria, Álava. Spain; Domingo Martínez: Intensive Care Unit, Hospital Universitario Virgen 
de Arrixaca, Crta. Madrid-Cartagena s/n, 30120 El Palmar, Murcia. Spain; Elena González: Intensive Care Unit, Hospital Universitario Virgen de La Luz, Hermanos Donantes de Sangre 1, 16002 Cuenca. Spain; M. Mar Cruz: Intensive Care Unit, Hospital Virgen de la Salud, Avda. Barber 30, 45004 Toledo. Spain; Songqiao Liu: Department of Critical Care Medicine, Zhongda Hospital, Southeast University, Nanjing, Jiangsu Province, 210009, China; Jesús Villar (not enrolling patients): Research Unit, Hospital Universitario Dr. Negrín, Barranco de la Ballena s/n, 35019 Las Palmas de Gran Canaria. Spain; Robert M. Kacmarek (not enrolling patients): Department of Respiratory Care, Massachusetts General Hospital, 55 Fruit St., Warren 1225, Boston, Massachusetts 01460, USA

\section{Author contributions}

RMK and JV contributed to the initial study concept and design. JV obtained funding for the study. JV, RMK, FA, SL, RM, CF, EG-H, DP, DM, CA, JMA, MS, and RLF contributed to the final study design. RMK, JV, CF, JMG-M participated in the first drafting of the manuscript. DP, FA, RS, SL, RM, JR-F, AV, CF, IM, RC, CEP, EG-H, QS, DP, DM, CA, JMA, and MS enrolled patients and participated in the data collection, data analysis or interpretation of data. JV, CF, JMG-M, and RMK are responsible for data analysis. JV, CF, RLF, and JMG-M had full access to all data in the study and take responsibility for the integrity of the data and the accuracy of the data analysis. All authors participated in the critical revision for intellectual content, and read and approved the final manuscript.

\section{Funding}

This trial was funded in part by a research grant from Maquet-Getinge Critical Care (Sölna, Sweden), and by academic grants from Instituto de Salud Carlos III, Madrid, Spain (CB06/06/1088, PI13/0119, PI16/0049), the European Regional Development's Funds (FEDER), and Asociación Científica Pulmón y Ventilación Mecánica (Las Palmas de Gran Canaria, Spain). NAVA catheters were provided free of cost by Maquet-Getinge. All researchers are independent of the funding bodies. None of the clinical investigators received any honorarium for enrolling patients.

\section{Compliance with ethical standards}

\section{Conflict of interest}

RM Kacmarek serves as a consultant for Medtronic and Orange Med Inc., and received research grants from Medtronic and Orange Med Inc and provided a web seminar on High Flow Nasal Cannulas and Ventilator Management of the Obese Patient for Nihon Kohden. J Villar received a research grant from Maquet. The rest of authors declare no potential conflict of interest.

\section{Ethical approval}

The study was approved by the referral Ethics Committee of Hospital Clínico Universitario, Valencia, Spain. We obtained ethics approval and negotiated contracts from participating hospitals before study initiation.

\section{Data sharing}

All data needed to evaluate the conclusions of this article are present and tabulated in the main text or in the ESM. This article is the result of an original randomized, controlled trial in patients with acute respiratory failure. For individual de-identified raw data that underlie the results reported in this article, please contact the corresponding authors.

\section{Publisher's Note}

Springer Nature remains neutral with regard to jurisdictional claims in published maps and institutional affiliations.

\section{Received: 24 April 2020 Accepted: 11 July 2020}

Published online: 6 September 2020

\section{References}

1. Colombo D, Cammarota G, Bergamaschi V, de Lucia M, Corte FD, Navalesi P (2006) Physiologic response to varying levels of pressure support and neurally adjusted ventilatory assist in patients with acute respiratory failure. Intensive Care Med 34:2010-2018
2. Beck J, Reilly M, Grasselli G et al (2009) Patient-ventilator interaction during neurally adjusted ventilatory assist in low birth weight infants. Pediatr Crit Care Med 65:663-668

3. Piquilloud L, Vignaux L, Bialais E et al (2011) Neurally adjusted ventilatory assist improves patient-ventilator interaction. Intensive Care Med 37:263-271

4. Spahija J, de Marchie M, Albert M et al (2010) Patient-ventilator interaction during pressure support ventilation and neurally adjusted ventilatory assist. Crit Care Med 38:518-526

5. Terzi N, Pelieu I, Guittet L et al (2010) Neurally adjusted ventilatory assist in patients recovering spontaneous breathing after acute respiratory distress syndrome: physiological evaluation. Crit Care Med 38:1830-1837

6. Blanch L, Villagra A, Sales B et al (2015) Asynchronies during mechanical ventilation are associated with mortality. Intensive Care Med 41:633-641

7. Colombo D, Cammarota G, Alemani M et al (2011) Observation in detecting patient-ventilator asynchrony. Crit Care Med 39:2452-2457

8. De Wit M, Miller KB, Green DA, Ostman HE, Gennings C, Epstein SK (2009) Ineffective triggering predicts increased duration of mechanical ventilation. Crit Care Med 37:2740-2745

9. Demoule A, Clavel M, Rolland-Debord C et al (2016) Neurally adjusted ventilatory assist as an alternative to pressure support ventilation in adults: a French multicenter randomized trial. Intensive Care Med 42:1723-1732

10. Ferreira JC, Diniz-Silva F, Moriya HT, Alencar AM, Amato MBP, Carvalho CRR (2017) Neurally adjusted ventilatory assist (NAVA) or pressure support ventilation (OSV) during spontaneous breathing trials in critically ill patients: a crossover trial. BMC Pulm Med 17:139

11. Hadfield DJ, Rose L, Reid F et al (2020) Neurally adjusted ventilatory assist vs. pressure support ventilation: a randomized controlled feasibility trial performed in patients at risk of prolonged mechanical ventilation. Crit Care 24:220

12. Liu L, Xu X, Sun Q et al (2020) Neurally adjusted ventilatory assist versus pressure support ventilation in difficult weaning. Anesthesiology 132:1482-1493

13. World Medical Association (2013) World Medical Association Declaration of Helsinki. Ethical Principles for Medical Research Involving Human Subjects. JAMA 310:2191-2194

14. Villar J, Belda J, Blanco J et al (2016) Neurally adjusted ventilatory assist in patients with acute respiratory failure: study protocol for a randomized controlled trial. Trials 15:500

15. Schulz KF, Altman DG, Moher D, For the CONSORT Group (2010) CONSORT 2010 statement: updated guidelines for reporting parallel group randomized trials. PLoS Med 7:e1000251

16. ARDS Definition Task Force, Ranieri VM, Rubenfeld GD, Thompson BT et al (2012) Acute respiratory distress syndrome: the Berlin Definition. JAMA 307:2526-2533

17. Maclntyre N (2007) Discontinuing mechanical ventilatory support. Chest 132:1049-1056

18. Schoenfeld DA, Bernard GR, Network ARDS (2002) Statistical evaluation of ventilator-free days as an efficacy measure in clinical trials of treatments for acute respiratory distress syndrome. Crit Care Med 30:1772-1777

19. Vincent JL, Moreno R, Takala J et al (1996) The SOFA (sepsis-related organ failure assessment) score to describe organ dysfunction/failure. Intensive Care Med 22:707-710

20. Moerer O (2012) Effort-adapted modes of assisted breathing. Curr Opin Crit Care 18:61-69

21. Kacmarek RM, Pirrone M, Berra L (2015) Assisted mechanical ventilation: the future is now! BMC Anesthesiol 15:110

22. Terzi N, Piquilloud L, Rozé H et al (2012) Clinical review; update on neutrally adjusted ventilatory assist-report of a round-table conference. Crit Care 16:225

23. Navalesi P, Longhini F (2015) Neurally adjusted ventilatory assist. Curr Opin Crit Care 21:58-64

24. Sinderby C, Navalesi P, Beck J et al (1999) Neural control of mechanical ventilation in respiratory failure. Nat Med 5:1433-1436

25. de la Oliva P, Schüffelmann C, Gómez-Zamora A, Villar J, Kacmarek RM (2012) Asynchrony, neural drive, ventilatory variability and COMFORT: NAVA versus pressure support in pediatric patients. A non-randomized cross-over trial. Intensive Care Med 38:838-846 
26. Barwing J, Pedroni C, Quintel M, Moerer O (2011) Influence of body position, PEEP, and intra-abdominal pressure on the catheter positioning for neutrally adjusted ventilatory assist. Intensive Care Med 37:2041-2045

27. Brander L, Leong-Poi H, Beck J et al (2009) Titration and implementation of neutrally adjusted ventilatory assist in critically ill patients. Chest 135:695-703

28. Figueroa-Casas JB, Connery SM, Montoya R, Dwivedi AK, Lee S (2014) Accuracy of early prediction of duration of mechanical ventilation by intensivists. Ann Am Thorac Soc 11:182-185
29. Kacmarek RM (2019) Noninvasive respiratory support for postextubation respiratory failure. Respir Care 64:658-678

30. Villar J, Martínez D, Mosteiro F et al (2018) Is overall mortality the right composite endpoint in clinical trials of acute respiratory distress syndrome? Crit Care Med 46:892-899 